\title{
Patient Monitoring and Abnormality Detection Along with an Android Application
}

\author{
A. P. Hridhya a , C. Periasamy a,, I. R. Rahul a \\ a Department of ECE, Vedavyasa Institute of Technology, Malappuram, Kerala, India.
}

*Corresponding Author cperiasamy@gmail.com (C Periasamy)

Received : 26 03 2019 Accepted : 08 05 2019

\begin{abstract}
The health related problems are becoming more and more critical. This necessitates the need for continuous monitoring of health parameters of the patient. The health monitoring electronic equipment's are not able to maintain health logs, analyzing the biomedical data obtained and assisting the patient and caregivers depending on the analysis. This project is intended to monitor the patient's bio-signals like Electro Cardio Gram (ECG), pulse rate, respiration rate and temperature. Suitable algorithms can be used to find out the abnormalities in these bio-signals. Today Electronic Patient Records (EPR) are confined to the hospital database and are available within the hospital information system. This project also aims to a modification of Electronic Patient Records depending on the real-time vital parameter feed. These updated EPR information instead of storing in the hospital database are uploaded through the internet and stored in a cloud system. The patient need not have to carry the medical records in the form of papers, films or Compact Disc (CD) while moving from hospital to hospital. The patient needs to carry only an android mobile phone. Using the android application, the medical records stored in the cloud can be retrieved. The physician can analyze the medical records of the patient either through personal computers or through the mobile phones with the help of android application.
\end{abstract}

Keywords:Abnormality detection algorithm, health monitoring, Threshold detection, respiration rate, cloud storage, Electronic Patient Record, Electro Cardio Gram

\section{Introduction}

The World Health Organization (WHO) estimated that about 17.5 million people died from Cardiovascular Disease (CVD) in 2012. The WHO also indicated that in 2014 , about $49 \%$ of deaths were related to heart failure and hence it is considered to be one of the most life-threatening CVDs. It is crucial to predict and avoid the occurrence of heart failure strokes [1]. It is necessary to continuously monitor patients and analyze the collected health data. The usual method of analysis of health data is based on comparing extracted health measurements to pre defined thresholds. Symptoms are detected when a measurement is higher or lower than a threshold. If it is able to detect the heart failure symptoms early, it is possible to make the prediction of heart failure strokes and consequently can avoid them $[1,2]$. So the most important task is to define accurate thresholds.

Existing remote health monitoring systems are based on the use of wearable sensors to extract health parameters [1 5]. Some of them can only monitor patient's data and do not analyze the collected data. This project aims to collect health parameters, processes collected data by executing analysis rules and find out if any abnormalities are present. The used analysis rules are typically based on comparing extracted health parameters to thresholds. They are not only a critical topic but also they deals with assembling, tracking and management of health records of several thousands of patients in a hospital unit. If they are managed properly and effectively it is possible to simplify the complete process of tracking a patient's health for the physician.

However, patient misidentification can have disastrous effects which may lead to medical errors and also increase the risk to the patient's safety. In a developing country like India, which has about 1.24 billion population; chances of patient record mismanagement are very large because the records are maintained through files and journals and are handled by minimally educated clerks who have insufficient medical knowledge. Because of a large number of human interactions among doctors, patients, nursing staff, there are chances for the operator's human errors to be introduced in the process [6]. This may leads to various problems such as wrong treatment given to a patient, faulty diagnosis etc. In most of the hospitals, patient identification is not implemented. For example in the file or 
journal based system, due to improper handling and mismanagement some crucial data may be misplaced or wrongly referenced and may be lost. Even if the data is maintained through a centralized server, there are chances for the manipulation of data due to the lack of biometric testing or validation of user's identity. Barcode based identification is also an effective technical method but it also fails to provide the updated real time information once the barcode is printed. These techniques can be implemented at the hospitals with minimum use to the user to be able to follow his medical chart. These issues clearly indicate the need for a cogent, user friendly and cost effective health management system which can also assist the user as well as the hospital administration. The medical records of the patient are stored in a cloud system. All the medical data which are stored in the online cloud system can be accessed through internet. For the ease of accessing the medical records, an android application is provided so that it can be accessed easily through mobile phones $[3,4,7]$.

\section{Existing System}

Medical records are the documents that explain all detail about the patient's history, clinical findings, diagnostic test results, pre and postoperative care, patient's progress and medication. If it is written correctly, this will support the doctor about the success of treatment. Proper record keeping in India is still in the primitive stages. Medical records are the one of the most important aspect on which almost every medico legal battle is won or lost. It is very important for the doctor to properly document the management of the patient under his care. Medical record keeping has evolved into a science [7]. The dispensability of most of the medical negligence claim is due to the quality of the medical records. Maintaining proper patient record is the only way for the doctor to prove that the treatment was carried out properly. Properly maintained medical records are the only source of the truth. They can be far more reliable than memory. The existing system for the bio signal monitoring consists of acquiring the signals from the body, processing them and extracting useful information from the signals. From these information abnormalities if present is identified. The results are given to the patient as hardcopy like paper films or as softcopy like CD. This information is stored in the hospital database if the patient has undergone an inpatient treatment as EPR [8]. But these EPR are not available to the patient when the patient prefers to consults another doctor or if a shifting of the hospital is done. The patient has no control over the medical records.

\section{Proposed System}

The proposed system consists of mainly three sections. 1)Bio signal measurement and processing part 2) Abnormality detection section 3) Android application. Body parameters like Electro Cardiogram, pulse rate, temperature and respiration rate are monitored. ECG measured using surface electrodes which are placed on the two arms and the right leg. The pulse rate is measured using IR sensor while the temperature is measured using temperature sensor. The respiration rate is measured using a condenser microphone.

Working The patient inserts the sensors in the body. They senses the signals from the body and convert them into electrical signals. The bio signals are given to AVR microcontroller. The output of the sensors can be directly connected to the microcontroller. If the output of the sensor is a continuous signal, it should be given to the ADC input. If the output is in the form of pulses, it can be connected to any I/O ports. The program for the processing of the signals is stored in the flash memory. After the processing of the signals in the microcontroller, the results are transferred to the computer through the serial port. A 16x2 LCD display is provided in the hardware side for displaying the measured parameters of the body during the time of measurement. For displaying the medical data on the computer screen a graphical user interface is provided. All the required parameters of the measured biosignals are displayed on the computer screen. Within the computer the algorithm for the abnormality detection is stored. The measured biological data obtained from the microcontroller is used by the algorithm to find out whether there are any abnormalities present in the biosignals. The results of the abnormality detection are also displayed in the computer screen.

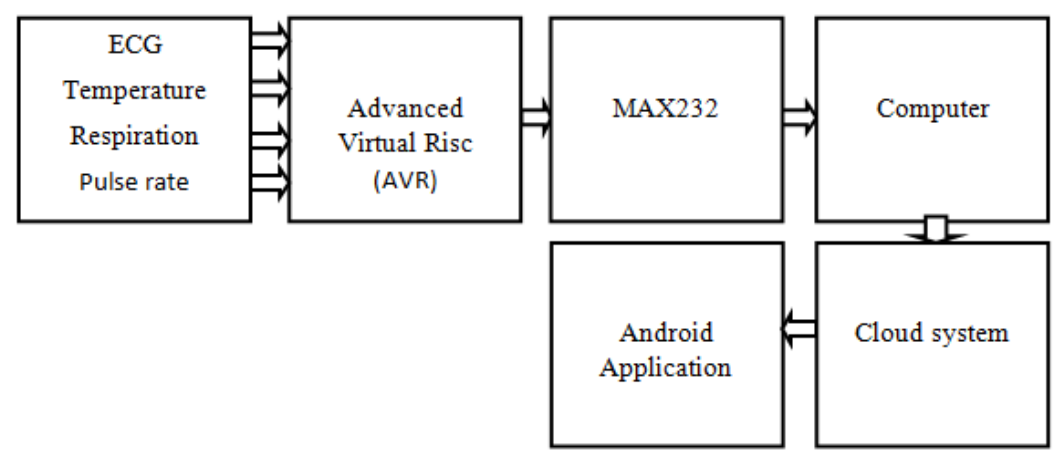

Figure1.Block diagram of the system 
All the measured body parameters are stored in a cloud system. The cloud storage systems allow accessing the data even in the event of power supply failure by one source. It also provides users with added protection and security for their stored data. For the easy and convenient retrieval of the patient information, an android application is also developed so that the medical records can be accessed through mobile phones. The medical records can also be accessed through personal computers using internet services.

ATmega16 ATmega16 is a low power microcontroller with 40 pin and is developed using CMOS technology. It has the property of low power consumption and high noise immunity. ATmega16 is based on AVR advanced RISC architecture. It is an 8 bit microcontroller. It is made of a single chip computer that comes with CPU, Read Only Memory (ROM), Random Access Memory (RAM), Electrically Erasable Programmable Read Only Memory (EEPROM), timers, counters, ADC and four 8 bit ports called PORT A, PORT B, PORT C, PORT D where each port consists of $8 \mathrm{I} / \mathrm{O}$ pins.. ATmega 16 is provided with two 8 bit timers and one 16 bit timer. These timers can be used as counters and can be optimized to count the external signal. The peripherals that are required to run automatic functions like ADC, analog comparator, Universal Synchronous Asynchronous Receiver/Transmitter (USART), Serial Peripheral Interface (SPI) functions are incorporated in this device. It is provided with $1 \mathrm{~KB}$ of static RAM which is volatile memory to store the information for short period of time and needs a constant power supply. A $16 \mathrm{~KB}$ of flash memory can store information for long period of time. It works on a maximum frequency of $16 \mathrm{MHz}$ where instructions are executed in one machine cycle.

ECG Recording For ECG recording, three surface electrodes are used. They are attached to the following sites to plot the ECG: each one on the inner surface of right and left forearm near the wrist and the third one on the inner surfaces of right leg near the ankles. The third one is to provide the reference. The electrodes are then connected to the circuit board using shielded cables for reducing noise interference. The ECG signal has to be amplified and filtered to get smooth desired signal without any noise [9]. The amplified and filtered signal is given to the ADC channel of the micro controller. The output is displayedUSB cable, or built into computer hardware, such as laptops[9].

Temperature measurement in the system using LM35 The LM35 series are precision integrated circuit temperature sensors. The output voltage of the sensor is linearly proportional to the Celsius (Centigrade) temperature. Over linear temperature sensors calibrated in ${ }^{\circ}$ Kelvin, the LM35 has an advantage that the user is not required to subtract a large constant voltage from its output to obtain convenient centigrade scaling. It is low cost by trimming and calibration at the wafer level.

Pulse rate measurement The number of times the heart beats per minute is the heart beat rate. The beat of the heart can be felt in any artery that lies close to the skin is called the pulse. A basic heartbeat sensor consists of a light emitting diode and a photo detector like a light detecting resistor or a photodiode. The heart beat pulses causes a variation in the flow of blood in different regions of the body. When a tissue is illuminated with a light source like the light emitted by the Light emitting Diode (LED), it either reflects (a finger tissue) or transmits the light (earlobe). Some of the incident light is absorbed by the blood and the transmitted or the reflected light is received by the light detector. The amount of light absorbed dependent on the blood volume in that tissue. The detector output will be in form of electrical signal and is proportional to the heart beat rate. This signal consist of a Direct Current (DC) signal relating to the tissues and the blood volume and the Alternating Current (AC) component synchronous with the heart beat and caused by pulsatile changes in arterial blood volume. The AC signal obtained is superimposed on the DC signal. So it is required to isolate that AC component as it is of prime importance. To achieve the process of getting the AC signal, the output from the detector is first filtered using a 2 stage high pass low pass circuit. By using a comparator circuit or using simple ADC the signal are converted into digital pulses. These digital pulses are given to an AVR microcontroller for calculating the heat beat rate.

Respiration rate measurement - The respiration rate shows the number of breaths a person takes per minute. It is calculated when a person is at rest and involves counting

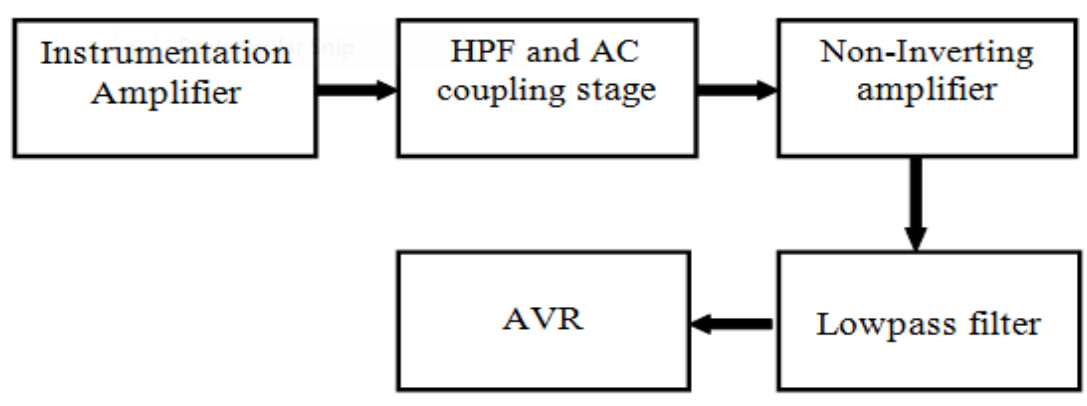

Figure2. Block diagram of ECG measurement system 
The number of breaths for one minute or by counting how many times the chest rises. Capacitor microphone uses a capacitance which varies with the incoming signal to generate the varying output voltage. The condenser or capacitor microphone depends on the changes in capacitance for its operation. CZN $15 \mathrm{E}$ Mic based Sound Sensor Module is a sound sensor module which provides a way to detect sound and is generally used for detecting sound intensity. The accuracy can be adjusted for the convenience of usage. It makes use of a microphone which supplies the input to an amplifier, peak detector and buffer. When a sound is detected, it is processed and an output signal voltage is sent to a microcontroller which performs necessary processing.

\section{Software Description}

Microcontroller programming is done in Embedded C using CodeVisionAVR Integrated Development Environment (IDE). The abnormality detection algorithm, serial communication with the computer and cloud storage is done in C\# using Visual Studio IDE.

CodeVisionAVR It is a C cross compiler, Integrated Program Development environment (IDE) and Automatic Program Generator designed for the Atmel AVR family of microcontrollers. The programs are designed to run under Windows 98, NT 4, 2000, XP and vista 32 bit operating systems. The C $\sim$ cross compiler implements all the elements of ANSI C language which are allowed by the AVR architecture, along with some features added to take the advantage of specificity if the AVR architecture and embedded system needs.

Visual Studio 2010 Call hierarchy of methods helps to view the flow of method calls to and from a particular method. The VS2010 IDE has a support for creating share point applications using its built in SharePoint Designer. It is provided with built in XSD Designer and Unified Modelling Language (UML) Designer. It has a built in UML designer that can be used to design UML diagrams from within the VS2010 IDE itself. The VS2010 IDE has a number of built -in project templates for designing and developing SharePoint applications using Visual Basic or $\mathrm{C \#}$ as the programming language.

Eclipse Eclipse is an IDE used for computer programming. It is a most widely used Java IDE. A base workspace and an extensible plug in system which is meant for customizing the environment are present in eclipse. Programming in eclipse is done mostly in Java and its primary use is for developing Java applications. Eclipse contains a discontinued Google provided plug in for the eclipse IDE called as Android Development Tools (ADT). It is designed to provide an integrated environment to build Android applications. ADT extends the abilities of Eclipse so that the developers set up new android projects, create an application user interface, add packages based on the android framework
Application Program Interface (API) etc. It is free download software

\section{Programming Languages}

The programming languages used in this project are Embedded $C$ and C\#. Embedded $C$ is used for the programming of AVR microcontroller. The abnormality detection and android development is done in C\# language.

Embedded $\boldsymbol{C} \sim$ Embedded $\mathrm{C}$ is a popular and most commonly used programming languages in the development of embedded systems. There are many programming languages used for developing embedded systems, Embedded C remains popular due to its efficiency, less development time and portability. CodeVisionAVR make use of embedded $\mathrm{C}$ for programming.

C\#-The name C\# can be pronounced as "C - Sharp". It runs on .Net framework. It is designed by Microsoft as an object oriented programming language. Different types of secured and robust applications can be developed using $\mathrm{C \#}$. It is designed for Common Language Infrastructure (CLI). It is a specification which describes executable code and runtime environment. C\# programming influenced by other programming languages like $\mathrm{C}++$, Java, Eiffel, Modula 3, Pascal etc. languages. The applications of $\mathrm{C \#}$ include window applications, web applications, distributed applications, web service applications, database applications etc.

\section{Results and Discussions}

This project is an effective tool for the monitoring bio signals like ECG, pulse rate and respiration rate. These parameters are so vital that the abnormalities in these values indicate early symptoms of certain diseases. The figure 3 shows the health monitoring system implemented in this project.

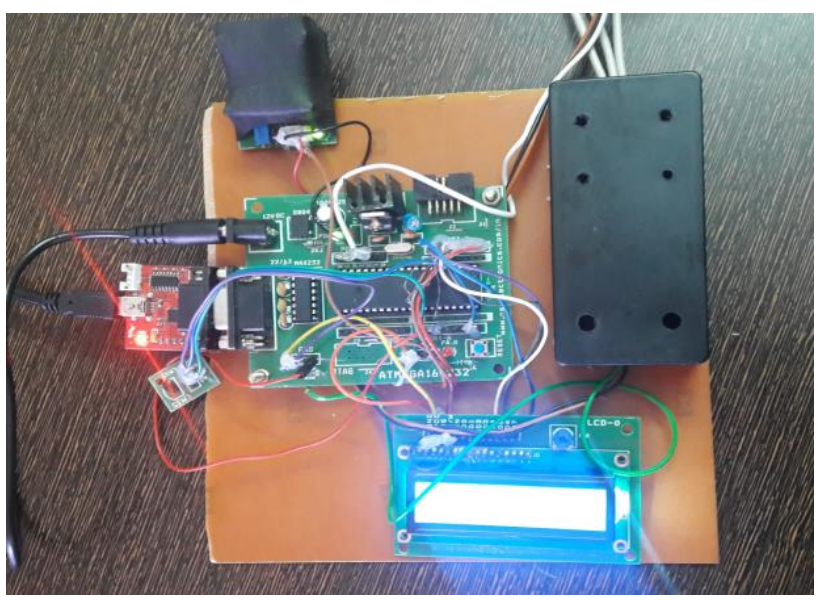

Figure3. Health monitoring system

The health monitoring is performed in two modes. In the first mode, temperature, pulse rate and respiration rates are displayed in the LCD display and these signals are transferred to the computer. Temperature is transmitted 
continuously to the computer while the values of respiration computer. A real time display of the all the biosignals rate and the pulse rate are updated in every 60 seconds. In measured and the abnormalities if any are displayed in the the second mode only ECG signal is transmitted. The AVR monitor.

microcontroller transmits the signals to the personal

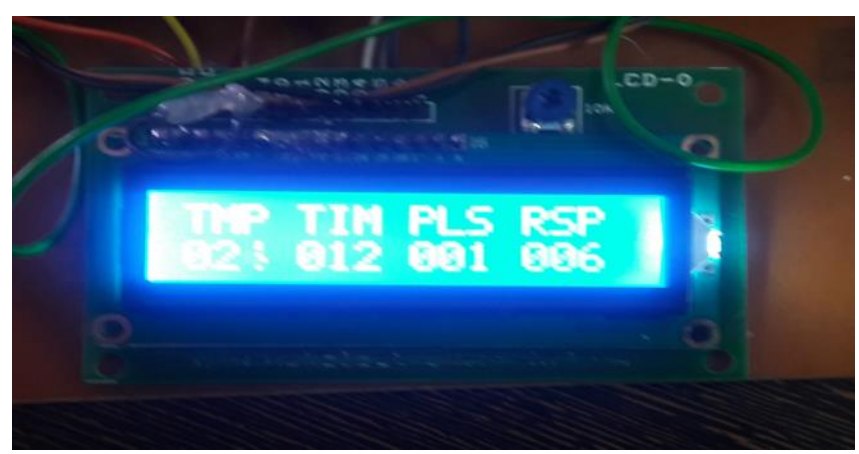

Figure 4.LCD Display

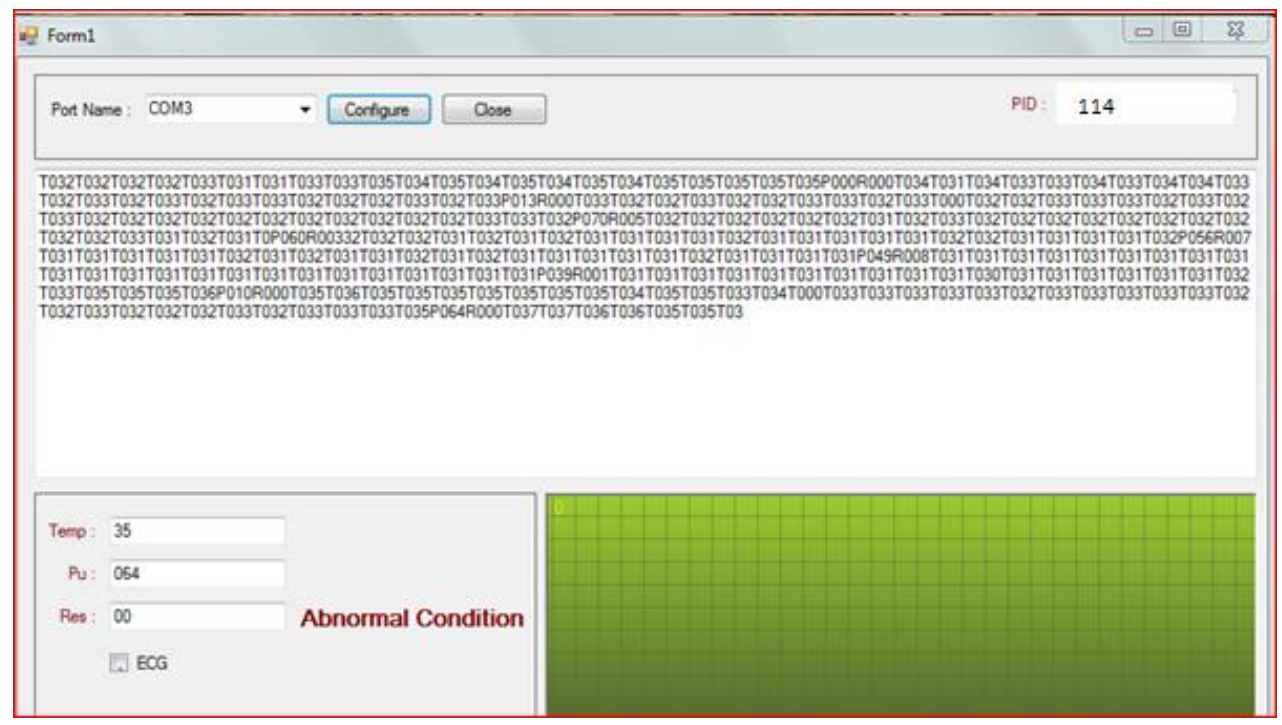

Figure 5. The display of the bio parameters in computer screen

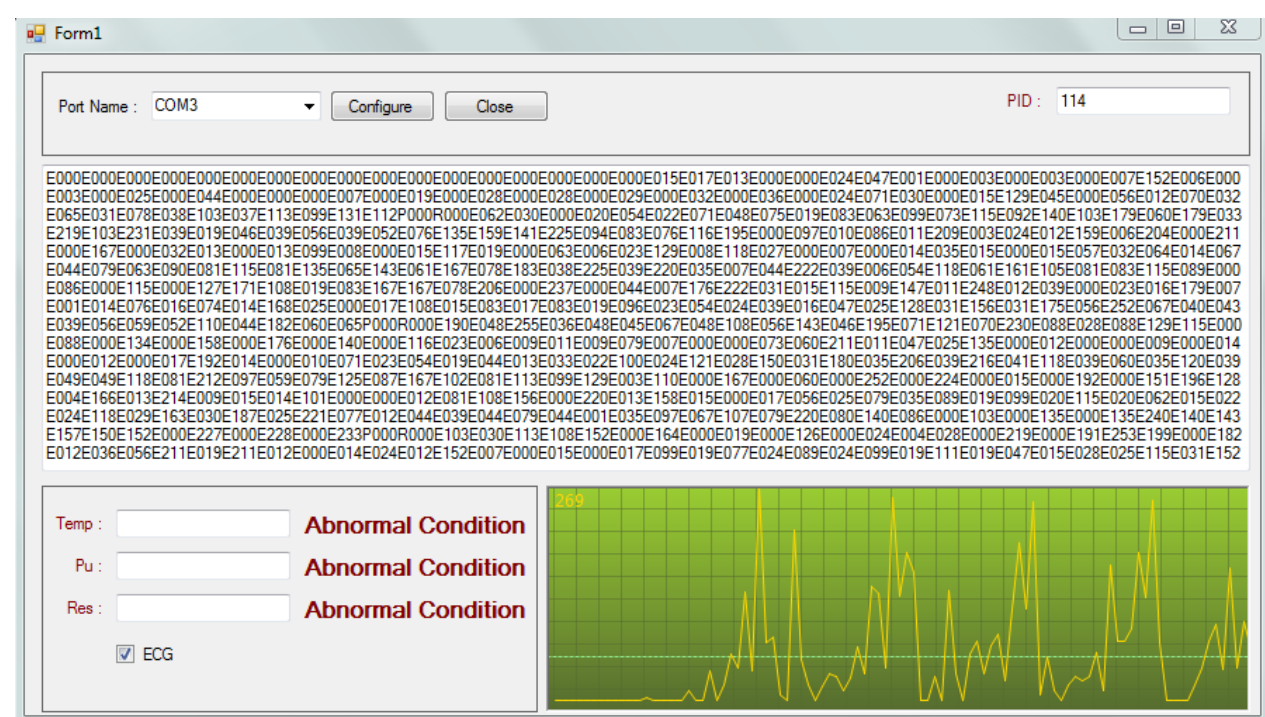

Figure 6.The display of ECG in the computer screen. 


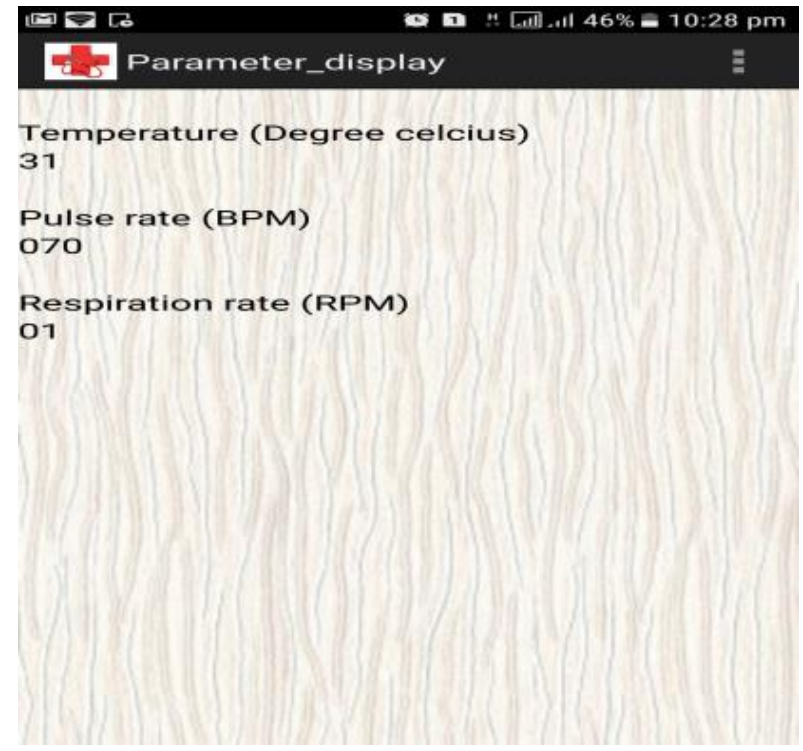

Figure 7.Display of the bio parameter view page in the health monitoring app

\section{Conclusion}

A major reason for the increase in the death rate is the lack of proper monitoring of the health data and improper analysis by the physician. This project proposes a low cost method for the monitoring of body parameters like ECG, temperature, respiration rate and pulse rate. The real time LCD display of bio signals is made at the monitoring side. The personal computer also acts a monitor for displaying bio parameters and ECG. Abnormalities detected are displayed in the monitor. The last recorded data is stored in the cloud system so that a patient can use his android mobile phone to retrieve the data when consulting the doctor. Thus this project provides a way for a paperless and more efficient medical recording system. This project proposes a model for the future medical recording and analysis system. As a future development more bio parameters can be included into the monitoring system. The abnormality detection algorithms can also be modified for the more accurate disease analysis. With further advancements the complete hardcopy based system can be replaced with the health monitoring system

\section{References}

[1] Afef Mdhaffar, Ismael Bouassida Rodriguez, Khalil Charfi, Leila Abid, and Bernd Freisleben, CEP4HFP: Complex Event Processing for Heart Failure Prediction, IEEE trans. nanobioscience, 16 (2017) 8.

[2] Myung kyung Suh, Chien An Chen, Jonathan Woodbridge, Michael Kai Tu, Jung In Kim, Ani Nahapetian, Lorraine S. Evangelista, and Majid Sarrafzadeh, A remote patient monitoring system for congestive heart failure, J. Med. Syst., 35(2011) 11651179 .

[3] O. Banos, C. Villalonga, M. Damas, P. Gloese koetter, H. Pomares, and I. Rojas, Physio Droid: Combining wearable health sensors and mobile devices for aubiquitous, continuous, and personal monitoring, Sci. World J., (2014) 490824.

[4] P. Kakria, N. K. Tripathi, and P. Kitipawang, A real time health monitoring system for remote cardiac patients using smartphone and wearable sensors, Int. J. Telemed. Appl., (2015) 8.

[5] U. Anliker, J.A. Ward, P. Lukowicz, G. Troster, F. Dolveck, M. Baer, F. Keita, E.B. Schenker, F. Catarsi, L. Coluccini, A. Belardinelli, D. Shklarski, M. Alon,E. Hirt, R. Schmid, M. Vuskovic, AMON: A wearable multiparameter medical monitoring and alert system, IEEE Trans. Inf. Technol. Biomed., 8 (2004) 415-427.

[6] A. Manashty, J. Light, and U. Yadav, Healthcare event aggregation lab (HEAL), a knowledge sharing platform for anomaly detection and prediction, in Proc. 17th Int. Conf. E-Health Netw., Appl. Services (Health Com), Boston, MA, USA, (2015) 648-652.

[7] Divyesh Dixit, Ankit Kalbande, K.M Bhurchandi, (2013), RFID Based Health Assistance \& Monitoring System through a Handmounted Embedded Device, IEEE - 4th ICCCNT, 31661.

[8] R. Pathak and V. Vaidehi, Complex event processing based remote health monitoring system, in Proc. 3rd Int. Conf. Eco Friendly Comput. Commun. Syst. (ICECCS), pp. 61-66.

[9] W. Y. Chung, S. Bhardwaj, A. Punvar, D. S. Lee, and R. Myllylae, A fusion health monitoring using ECG and accelerometer sensors for elderly persons at home, in Proc. 29th Annu. Int. Conf. IEEE Eng. Med.Biol. Soc. (2007) 3818-3821.

\section{About The License}

(C) 2019 The Authors. This work is licensed under a Creative Commons Attribution 4.0 International License which permits unrestricted use, provided the original author and source are credited. 